\title{
An integral equation formulation for the diffraction from convex plates and polyhedra
}

\author{
Andreas Asheim U. Peter Svensson
}

Report TW610, March 2012

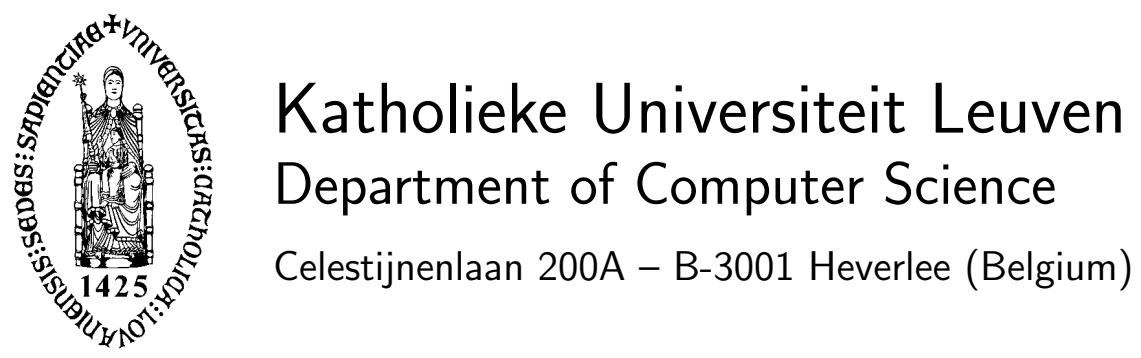




\title{
An integral equation formulation for the diffraction from convex plates and polyhedra
}

\author{
Andreas Asheim U. Peter Svensson \\ Report TW610, March 2012 \\ Department of Computer Science, K.U.Leuven
}

\begin{abstract}
A new formulation of the problem of scattering from obstacles with edges is presented. The formulation is based on decomposing the field into geometrical optics and edge diffraction components, as opposed to the usual incident and scattered wavefields used for BEM and FEM methods. A secondary-source model is available for edgediffraction, which is extended to handle multiple diffraction of all orders. We show that the diffraction component can be represented by the solution of an integral equation formulated on pairs of edge points, so that the total field can be evaluated as as sum of geometrical optics and this diffraction component. Numerical experiments demonstrates the validity of the approach for wavenumbers down to 0 .
\end{abstract}

Keywords : Scattering, diffraction, integral equations, convex plates, convex polyhedra

MSC : Primary : 65R20, Secondary : 45B05, 78A05, 78A45. 


\title{
An integral equation formulation for the diffraction from convex plates and polyhedra
}

\author{
Andreas Asheim* U. Peter Svensson ${ }^{\dagger}$
}

March 5, 2012

\begin{abstract}
A new formulation of the problem of scattering from obstacles with edges is presented. The formulation is based on decomposing the field into geometrical optics and edge diffraction components, as opposed to the usual incident and scattered wavefields used for BEM and FEM methods. A secondary-source model is available for edge-diffraction, which is extended to handle multiple diffraction of all orders. We show that the diffraction component can be represented by the solution of an integral equation formulated on pairs of edge points, so that the total field can be evaluated as as sum of geometrical optics and this diffraction component. Numerical experiments demonstrates the validity of the approach for wavenumbers down to 0 .
\end{abstract}

\section{Introduction}

Fast and accurate simulation of wave scattering phenomena is of importance in a wide range of application areas, e.g. medical imaging, geophysical exploration, radar and sonar technology, architectural acoustics, etc. A number of different approaches are possible for solving such wave scattering problems. For most numerical approaches, like e.g. FEM and BEM, one solves for the scattered field $u^{s}$, given the incident field $u^{i}$. Classical high frequency asymptotic methods like the geometrical optics approximation

\footnotetext{
*Departement Computerwetenschappen, Celestijnenlaan 200a, University of Leuven, 3001 Heverlee, Belgium (andreas.asheim@cs. kuleuven.be). Supported by the Norwegian Research Council's Magne S. Espedahl Fellowship.

${ }^{\dagger}$ Acoustics Research Centre, Department of Electronics and Telecommunications, Norwegian University of Science and Technology, 7491 Trondheim, Norway (svensson@iet.ntnu.no)
} 
represent the total field in terms of a direct and reflected field, plus a rest, often referred to as the diffracted field. This is notably different from existing numerical approaches. Whereas the scattered field $u^{s}$ will approach zero everywhere as the wavenumber $k \rightarrow 0$, the diffracted field approaches zero as $k \rightarrow \infty$. The geometrical approach is, in being high frequency asymptotic, the natural setting for high frequency scattering problem, and similarly the incident/scattered field approach must be seen as the natural setting for low frequencies. The latter is however the most widely used for numerical solvers, also for moderately high frequency problems. The reason for this is mainly because exact representations of the diffracted field is only known for a few canonical shapes. For most practical problems asymptotic solution methods with limited/asymptotic accuracy, like the geometric theory of diffraction (GTD) [11] or uniform theory of diffraction (UTD) [12], are used.

Recently, a number of approaches for taking geometrical components into account in numerical solvers have been suggested. Notably, so-called phaseextraction techniques $[4,6,7,1]$ imply factoring geometric components out and solving for a rest. This leads to methods that are particularly efficient for high frequency scattering problems. A similar approach applicable to $2 \mathrm{D}$ scatterers with edges, taken in [5], involves solving for the amplitude of geometric components and phase factors obtained from edge diffraction, and solving for the rest. A review of such methods can be found in [17]. Although such methods go a long way in separating the geometrical and diffracted fields, none of them take the full step.

The decomposition of the wavefield into geometrical components and diffraction components is well-established for the scattering from a single infinite wedge with rigid (Neumann) and soft (Dirichlet) boundary conditions. Various formulations of the exact solution for the canonical case of an infinite wedge are known; in the frequency-domain $[14,16]$, and time-domain $[2,15,24]$, as well as the case of electromagnetic problems[18]. For polyhedra and non-straight edges, however, multiple-order diffraction waves will be generated and these have been studied to much less degree. Formulations for such multiple-order diffraction waves have been suggested as a cascading of first-order diffraction waves [15, 24], also for high-frequency asymptotic methods like the UTD [9] and others [25]. The validity of this cascading is well-established in the high-frequency limit but its validity in the lowfrequency limit has not been demonstrated rigorously. Of special interest is the so-called slope diffraction. This concept was described by Keller, [11], but the term slope diffraction was introduced later. Slope diffraction implies that an edge will generate a diffracted wave if it is hit by an incident sound wave with amplitude 0, as long as there is a non-zero gradient which hits 
the edge. Such a non-zero gradient is particularly prominent for a hole, or a slit, in a thin infinite screen with a Neumann boundary condition. Correspondingly, the scattering from objects with a Dirichlet boundary condition involves slope diffraction, cf. Babinet's Principle. As was pointed out by Summers, [20], the methods in [15, 24] ignore slope diffraction and therefore give significant errors for those special cases with holes in thin screens. However, that particular type of slope diffraction vanishes when convex scatterers are studied.

In this work we explore how, for a class of 3D scatterers with edges, equations for the diffracted field can be built up from an analytical representation of the diffracted field for a single wedge. We study multiple-order diffraction waves more in detail for the case of convex polyhedra, and flat, convex plates with possibly curved edges, and then show that for such scattering problems, a representation for the diffracted field can be given in terms of a solution to an integral equation whose unknown is a function defined on pairs of edge points. This is notably different from methods due to Chandler-Wilde, Langdon et al. [5] for 2D scatterers, and Hewett et al. [17] for 3D screens (plates), that are based on separating the geometric optics and diffracted fields in classical boundary integral equations. The validity of the formulation is demonstrated by comparing with known low-frequency asymptotics and heuristics, which shows a performance which would a-priori not be expected by a method based on geometrical optics. For higher frequencies, the validity essentially follows from the validity of diffraction cascade.

The paper is built up as following: We will first present the theory of wedge diffraction in $\S 2$, before in $\S 3$ we derive the integral formulation for the diffraction from plates and polyhedra. We treat the special case of axisymmetrical scattering from a circular disk more in detail. In $\S 4$ we present numerical experiments.

\section{Scattering of scalar waves}

The time-harmonic scattering of scalar waves by an obstacle $\Omega$ is formulated through the Helmholtz equation: Given an incoming wavefield $u^{i}$ we seek a scattered wavefield $u^{s}$ satisfying a Sommerfeld radiation condition such that the total field $u=u^{i}+u^{s}$ satisfies

$$
\Delta u+k^{2} u=0 \quad x \in \mathbb{R}^{3} \backslash \Omega .
$$






Figure 1: Geometrical optics: The field reflected from a polyhedron face is computed as the field from a virtual source, with opposite polarity in the Dirichlet case, $d=d^{\prime}$, viewed through the polyhedron face.

This problem is well posed provided that boundary conditions on $\partial \Omega$ are supplied. Typically these are of Dirichlet type, $\left.u\right|_{\partial \Omega}=0$, or Neumann type, $\left.\frac{\partial u}{\partial n}\right|_{\partial \Omega}=0$. The geometrical optics approximation to the field is an asymptotic approximation valid in illuminated regions of convex obstacles. Geometrical optics operates with rays, and as such it gives rise to familiar concepts like reflections and shadows. For scatterers with flat faces, the reflected field in geometrical optics is calculated in a straightforward manner using the method of images (see Figure 1). We will refer to a decomposition of the total field $u$ in terms of direct, reflected, and diffracted fields,

$$
u(x)=u_{\text {direct }}(x)+u_{\text {reflected }}(x)+u_{\text {diffracted }}(x),
$$

where the first two terms are given from geometrical optics.

\subsection{Diffraction from a finite wedge}

Considering a point source at $S, u^{i}(x)=\mathrm{e}^{\mathrm{i} k r_{S, x}} / r_{S, x}$, where $r_{a, b}$ denotes the Euclidean distance between the points $a$ and $b$. The diffraction components for the scattering by an infinite wedge are well known $[2,16]$. The diffraction wave can in this case be written as a line integral along the edge, with an integrand which includes spherical divergence factors as well as analytical directivity factors, and with the position along the physical wedge as integration variable, either in the time-domain [24] or frequency domain [23]. A 


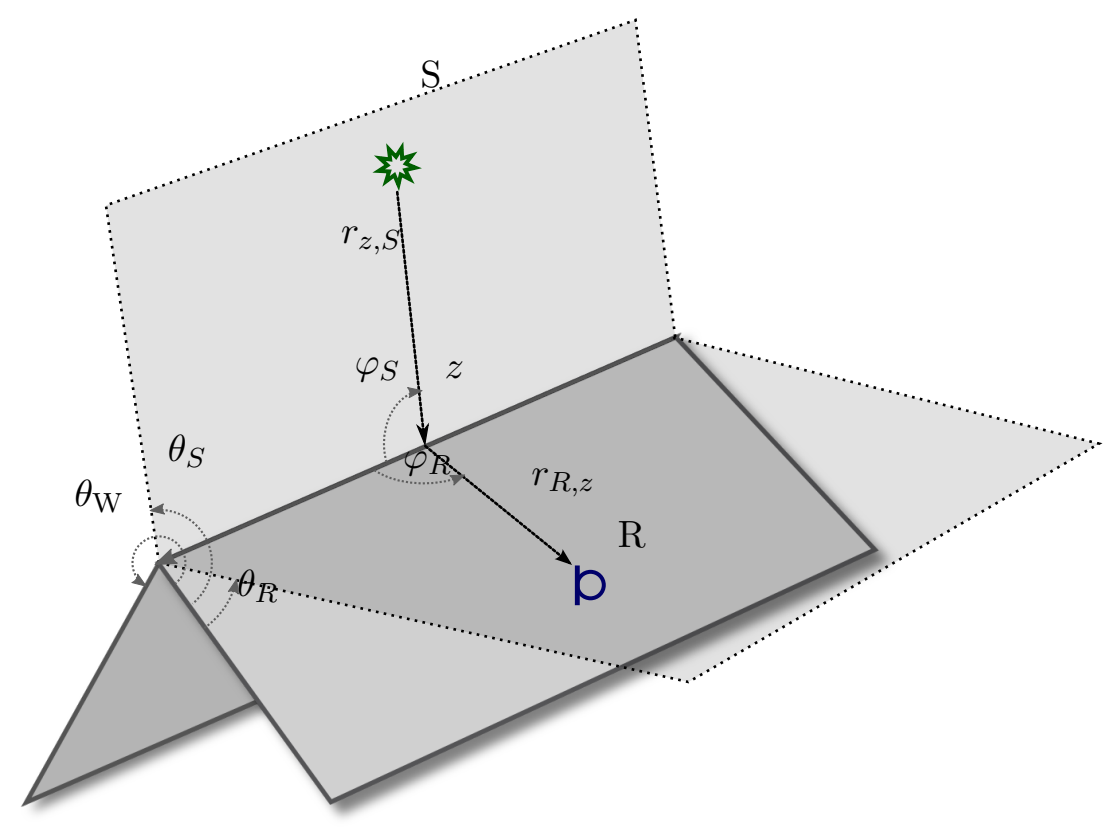

Figure 2: A single wedge.

finite wedge is handled simply by setting the integration range according to the physical finite edge. The diffracted field at a receiver position $R$ is thus expressed by the integral,

$$
u_{\text {diffracted }}(R)=-\frac{\nu}{4 \pi} \int_{z_{1}}^{z_{2}} \frac{\mathrm{e}^{\mathrm{i} k r_{R, z}}}{r_{R, z}} \frac{\mathrm{e}^{\mathrm{i} k r_{z, S}}}{r_{z, S}} \beta(R, z, S) \mathrm{d} z .
$$

The function $\beta(R, z, S)$, which will be referred to as a directivity function, is in the Neumann case given as

$$
\beta(R, z, S)=\sum_{i=1}^{4} \frac{\sin \left(\nu \phi_{i}\right)}{\cosh (\nu \eta)-\cos \left(\nu \phi_{i}\right)},
$$

where the angles $\phi_{i}$ are

$$
\begin{array}{ll}
\phi_{1}=\pi+\theta_{S}+\theta_{R}, & \phi_{2}=\pi-\theta_{S}+\theta_{R}, \\
\phi_{3}=\pi-\theta_{S}-\theta_{R}, & \phi_{4}=\pi+\theta_{S}-\theta_{R} .
\end{array}
$$


The Dirichlet case is handled simply by inserting a factor $(-1)^{i}$ in the summation, Eq. (3) [3]. We will however in the following concentrate in the Neumann case since, as mentioned in the introduction, multiple diffraction at an object with a Dirichlet boundary condition involves slope diffraction. The angles $\theta_{S}$ and $\theta_{R}$ refer to the angle between the planes containing the edge and $S$, and $R$ and a reference wedge plane, respectively, as illustrated in Figure 2. The function $\eta$ is an auxiliary function,

$$
\eta=\cosh ^{-1} \frac{\cos \varphi_{S} \cos \varphi_{R}+1}{\sin \varphi_{S} \sin \varphi_{R}}
$$

where the angles $\varphi_{S}$ and $\varphi_{R}$ refer to the angle between the edge and $S$ and $R$, respectively, which is also illustrated in Figure 2 . The fact that $\eta$, Eq. (4), does not include any distance motivates why $\beta$ can be called a directivity function for secondary edge sources. We will in the following refer to the pairs $\theta_{S}$ and $\varphi_{S}$, and $\theta_{R}$ and $\varphi_{R}$ as the source and receiver angles respectively. The quantity $\nu$ is the so-called wedge index which is determined by the angle $\theta_{W}$ (see Fig. 2) formed by the two wedge planes,

$$
\nu=\frac{\pi}{\theta_{W}} \text {. }
$$

\subsection{Multiple diffraction components}

The expression for the directivity $\beta$ can be directly applied to curved edges as well as straight edges, in which case the source and receiver angles are defined relative to the edge tangent, and are moreover functions of the position along the edge. Generalizing Eq. (2) to curved edges is then done in a straightforward manner by instead considering the integral as a line integral [24]. For an edge given as the curve $\Gamma$, we get

$$
u_{\text {diffracted }}^{(1)}(R)=-\frac{\nu}{4 \pi} \int_{\Gamma} \frac{\mathrm{e}^{\mathrm{i} k r_{R, z}}}{r_{R, z}} \frac{\mathrm{e}^{\mathrm{i} k r_{z, S}}}{r_{z, S}} \beta(R, z, S) \mathrm{d} s_{z} .
$$

Note however that a curved edge will necessarily generate higher-order diffraction terms, and this expression will only constitute the first order diffraction term (hence the superscript ${ }^{(1)}$ ). Like a curved edge must give higher order diffraction effects, so does a finite edge, since a finite edge is presumably connected to other edges. These higher-order diffraction terms, which can be expressed as a cascade of first-order diffraction, are all added together to give the total diffracted field, which is necessarily smooth, i.e., without the discontinuities that appear in a geometrical optics approximation. 


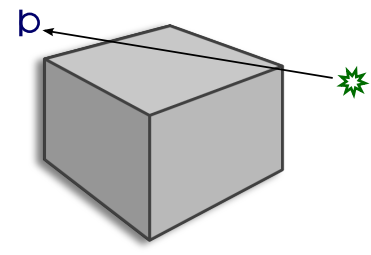

(a)

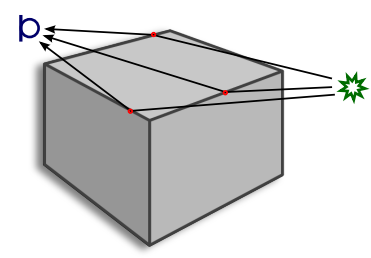

(c)

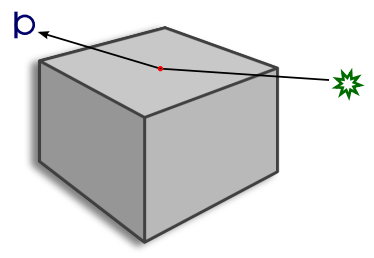

(b)

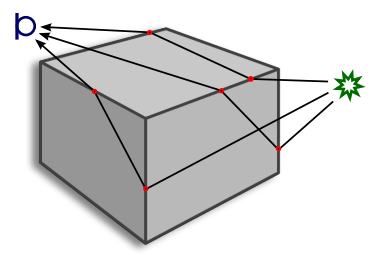

(d)

Figure 3: The decomposition of the wavefield: a) Direct, b) Specular reflection, c) First order diffraction, d) Second order diffraction.

The diffraction term comprises diffraction of all orders. Let the $n$-th order diffraction term be denoted $u_{\text {diffracted }}^{(n)}$, then

$$
u_{\text {diffracted }}=\sum_{n=1}^{\infty} u_{\text {diffracted }}^{(n)} .
$$

This approach can be illustrated as in Fig. 3, where the direct, reflected, first- and second-order diffraction components for the scattering by a polyhedron are indicated. In the illustration, single points along the edges are marked, but the entire edge contributes for each path. For the first order diffraction term, $u_{\text {diffracted }}^{(1)}$, the line integral form (2) is used. Denoting by $\Gamma$ the edges of the scatterer, we have

$$
u_{\text {diffracted }}^{(1)}(R)=-\frac{1}{4 \pi} \int_{\Gamma} \nu_{z} V_{R, z} V_{z, S} \frac{\mathrm{e}^{\mathrm{i} k r_{R, z}}}{r_{R, z}} \frac{\mathrm{e}^{\mathrm{i} k r_{z, S}}}{r_{z, S}} \beta(R, z, S) \mathrm{d} s_{z} .
$$

Here $V_{a, b}$ is a visibility factor which is one if $a$ is visible from $b$ and zero otherwise, and $\nu_{z}$ denotes the wedge index of the edge containing $z$.

For the second- and higher-order diffraction terms, previous presentations have used separate second- and higher-order integrals for each order 
[24]. Thus the second order field would be represented by the double integral,

$$
\begin{aligned}
u_{\text {diffracted }}^{(2)}(R)= & \frac{1}{2}\left(-\frac{1}{4 \pi}\right)^{2} \int_{\Gamma} \int_{\Gamma} \nu_{z_{1}} \nu_{z_{2}} V_{R, z_{1}} V_{z_{1}, z_{2}} V_{z_{2}, S} \\
& \frac{\mathrm{e}^{\mathrm{i} k r_{R, z_{1}}}}{r_{R, z_{1}}} \frac{\mathrm{e}^{\mathrm{i} k r_{z_{1}, z_{2}}}}{\mathrm{e}_{z_{1}, z_{2}}} \frac{\mathrm{i}_{z_{2}, S}}{r_{z_{2}, S}} \beta\left(R, z_{1}, z_{2}\right) \beta\left(z_{1}, z_{2}, S\right) \mathrm{d} s_{z_{1}} \mathrm{~d} s_{z_{2}} .
\end{aligned}
$$

Here, a a factor $1 / 2$ is introduced, since only half of the total field propagating along a surface diffracts at the second edge, as is pointed out in e.g. [12]. This higher integral dimensionality for each diffraction order leads to an exponential growth in computational load for increasing diffraction orders. In the following we will propose to rather work with the sum of the multiple diffraction components of order $2, \ldots, \infty$, which lends itself to more efficient treatment.

\section{A multiple diffraction integral equations}

Here, an integral equation will be presented, whose solution represents, along with $u_{\text {diffracted }}^{(1)}$, the diffracted field for the scattering from convex plates and polyhedra. Limiting ourselves to convex shapes with plane faces simplifies the derivation, since all visibility factors and edge indices $\nu_{z}$ will be constant along edges. First we shall treat in some detail the case of a flat plate, in

which case the visibility factors are all constant one. Then the analogous case of a convex polyhedron case follows.

\subsection{The case of a convex plate}

Consider a thin plate which is convex in shape (that is, without indents). The edge of the plate is denoted $\Gamma$. Focusing on the first order diffraction term, we have from the above discussion that the first order diffraction takes the form of a single integral along the edge of the plate like in equation (5),

$$
u_{\text {diffracted }}^{(1)}(R)=-\frac{1}{8 \pi} \int_{\Gamma} \frac{\mathrm{e}^{\mathrm{i} k r_{R, z}}}{r_{R, z}} \frac{\mathrm{e}^{\mathrm{i} k r_{z, S}}}{r_{z, S}} \beta(R, z, S) d s_{z} .
$$

No visibility factors are needed in this case; the wedge index in this case is a constant $\nu_{z}=1 / 2$. A detail must however be noted for the following: For higher order diffraction, a diffracted wave traveling between two points on the edge of a plate can take two different routes, i.e., over and under the 
plate. Because of this symmetry, a factor 2 must be introduced for scattering orders 2 and above. This factor is however canceled by the $1 / 2$ factor, as discussed in $\S 2.2$.

It is now instructive to realise the direct physical interpretation of the different factors in the integral (9): $\mathrm{e}^{\mathrm{i} k r_{z, S}} / r_{z, S}$ is the field experienced at the point $z$ due to the source at $S$ via spherical spreading. Likewise $\mathrm{e}^{\mathrm{i} k r_{R, z}} / r_{R, z}$ is the field experienced at $R$ due to a source at $z$. However, the strength of this source, which is located at the edge, as experienced at $R$, depends on angles between $R, z$ and $S$. This is what is encoded in the directivity function. This angular dependence of the field strength on both the incoming and the exiting angle explains why we in the following can't formulate using simple points, but rather need to consider pairs of points along the edge in order to formulate diffraction of all orders.

What will in the following be referred to as a source term is a quantity, $q_{0}\left(z_{1}, z_{2}\right)$, which is seen as an equivalent edge source strength at $z_{2}$ due to the field diffracted from $S$ (via $z_{2}$ ) in the direction of $z_{1}$,

$$
q_{0}\left(z_{1}, z_{2}\right)=-\frac{1}{8 \pi} \frac{\mathrm{e}^{\mathrm{i} k r_{z_{2}, S}}}{r_{z_{2}, S}} \beta\left(z_{1}, z_{2}, S\right) .
$$

The meaning of this quantity should be apparent when we define a propagation operator,



It can now be verified that $\mathcal{I}_{\text {prop. }}\left[q_{0}\right]=u_{\text {diffracted }}^{(2)}$, cf. Eq. (8).

Now define an integral operator that operates on functions in the class $\Gamma^{2} \rightarrow \mathbb{C}$

$$
\mathcal{I}[q]\left(z_{1}, z_{2}\right):=-\frac{1}{8 \pi} \int_{\Gamma} q\left(z_{2}, z\right) \frac{\mathrm{e}^{\mathrm{i} k r_{z_{2}, z}}}{r_{z_{2}, z}} \beta\left(z_{1}, z_{2}, z\right) d s_{z} .
$$

From the above discussion, it should be clear that this operator transforms the function $q\left(z_{1}, z_{2}\right)$, the equivalent source strength at $z_{2}$ radiating in the direction of another edge point $z_{1}$, to the first order diffracted field in the direction of $z_{1}$ due to the sum of the contribution from all edge sources, via $z_{2}$. (see illustration in Figure 4). Note that the directivity-function is now evaluated for triplets of edge-points, which will lead to simplifications. In particular it is shown in [24], for the rigid case,

$$
\beta\left(z_{R}, z, z_{S}\right)=\frac{4}{\cosh \frac{\eta}{2}}=4 \frac{\sqrt{\sin \varphi_{z_{R}} \sin \varphi_{z_{S}}}}{\cos \frac{\varphi_{z_{R}}-\varphi_{z_{S}}}{2}}, \quad z_{R}, z_{S} \in \Gamma .
$$




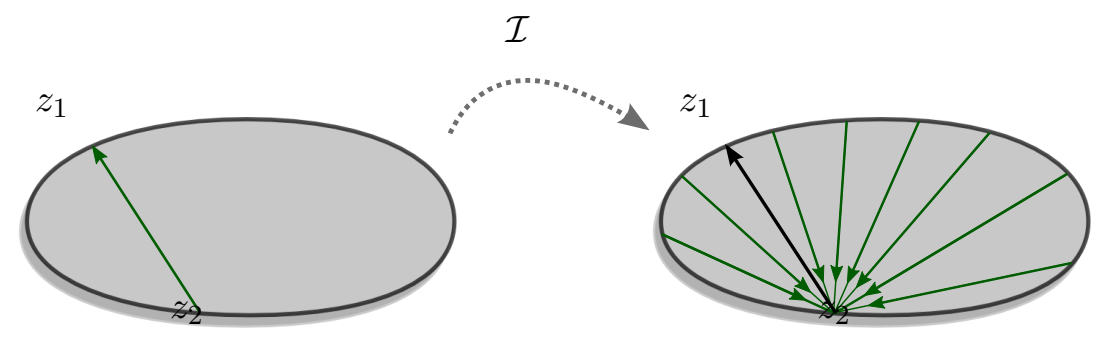

Figure 4: The action of the integral operator (11).

In the case of the propagation, two of the three points are on the edge, and in this case,

$$
\beta_{i}\left(R, z, z_{S}\right)=4 \frac{\cosh \frac{\eta}{2} \cos \frac{\theta_{R}}{2}}{\cosh ^{2} \frac{\eta}{2}-\sin ^{2} \frac{\theta_{R}}{2}} .
$$

Because of symmetry, the same expression holds for $\beta\left(z_{R}, z, S\right)$, i.e., the source term.

We can now extract diffraction of all orders, as experienced at edge points. To get to the next order diffraction we simply apply the integral operator,

$$
q_{1}\left(z_{1}, z_{2}\right)=\mathcal{I}\left[q_{0}\right]\left(z_{1}, z_{2}\right),
$$

which will represent the equivalent source strength for second order diffracted field at any edge point $z_{2}$ in the direction of $z_{1}$ due to the source $S$. Similarly, higher orders become,

$$
\begin{gathered}
q_{2}\left(z_{1}, z_{2}\right)=\mathcal{I}\left[q_{1}\right]\left(z_{1}, z_{2}\right)=\mathcal{I}^{2}\left[q_{0}\right]\left(z_{1}, z_{2}\right), \\
\vdots \\
q_{n}\left(z_{1}, z_{2}\right)=\mathcal{I}\left[q_{n-1}\right]\left(z_{1}, z_{2}\right)=\mathcal{I}^{n}\left[q_{0}\right]\left(z_{1}, z_{2}\right) .
\end{gathered}
$$

Evaluating the diffracted field of different order is now simply an application of the propagation operator, $u_{\text {diffracted }}^{(n+2)}=\mathcal{I}_{\text {prop. }}\left[q_{n}\right]$. Summing diffraction terms of all orders yields, using the Neumann series,

$$
\begin{aligned}
q\left(z_{1}, z_{2}\right) & :=\sum_{i=0}^{\infty} q_{i}\left(z_{1}, z_{2}\right) \\
& =\sum_{i=1}^{\infty} \mathcal{I}^{i}\left[q_{0}\right]\left(z_{1}, z_{2}\right)=(I d-\mathcal{I})^{-1}\left[q_{0}\right]\left(z_{1}, z_{2}\right) .
\end{aligned}
$$


where $I d$ is the identity operator. In other words, assuming the quantity $q$ exists it must satisfy an equation of the form

$$
q\left(z_{1}, z_{2}\right)=q_{0}\left(z_{1}, z_{2}\right)+\mathcal{I}[q]\left(z_{1}, z_{2}\right) .
$$

The total diffracted field at the receiver position $\mathrm{R}$ is evaluated trough the formula

$$
u_{\text {diffracted }}(R)=u_{\text {diffracted }}^{(1)}(R)+\mathcal{I}_{\text {prop. }}[q](R) .
$$

Here we should point out a couple of things: First, note that $R$ never appears in the computation of $q$. This implies that once $q$ is computed for one source position, or a superposition of sources, the field at any receiver position may be evaluated by evaluating the double integral (10). This is the same two-stage computational approach as the numerical solution of the Kirchhoff-Helmholtz integral equation; the boundary element method. Secondly, writing out the equation, we immediately see that it bears little resemblance to classical integral equations in scattering theory. The closest relative is probably the rendering equation, known from computer graphics [8].

In case we are interested in a plane wave incidence of the form $\mathrm{e}^{\mathrm{i} k d^{T} x}$, $\|d\|=1$, the only difference to the method is a change in the source term,

$$
q_{0}\left(z_{1}, z_{2}\right)=-\frac{1}{8 \pi} \mathrm{e}^{\mathrm{i} k d^{T} x} \beta\left(z_{1}, z_{2}, S_{\infty}\right) .
$$

The source angle are in this case computed naturally relative to the propagation direction given by $d$. The same modification must be done for the computation of $u_{\text {diffracted }}^{(1)}(R), u_{\text {direct }}(R)$, and $u_{\text {reflected }}(R)$.

\subsection{The case of convex polyhedra}

For the case of convex polyhedra the derivation is completely analogous to what is described for the thin plate scattering, with the small but important modification that the factor of 2 due to over/under plate symmetry vanishes. We shall here briefly state the result.

The first order diffraction term in this case is given in equation (7). Following the lead from the case of the thin plate we define an integral operator which now includes visibility factors as well as the wedge index,

$$
\mathcal{I}[q]\left(z_{1}, z_{2}\right):=-\frac{1}{8 \pi} \int_{\Gamma} q\left(z_{2}, z\right) \frac{\mathrm{e}^{\mathrm{i} k r_{z_{2}}, z}}{r_{z_{2}, z}} \nu_{z_{2}} V_{z_{1}, z_{2}} V_{z_{2}, z} \beta\left(z_{1}, z_{2}, z\right) d s_{z} .
$$




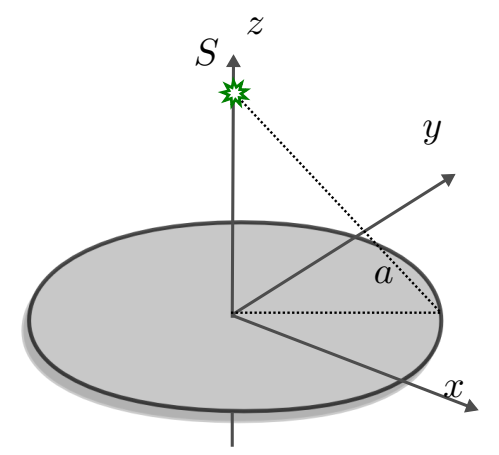

Figure 5: The axis-symmetric case: a circular disk of radius $a$ with source on the disk axis.

Again, this is an integral operator that takes $q\left(z_{1}, z_{2}\right)$, the function that assigns equivalent source strength at $z_{2}$, in the direction of $z_{1}$, and transforms it into the first order diffracted field at $z_{2}$ in the direction of $z_{1}$ due to the integral over contributions from all edge sources that pass via $z_{2}$. Using as source

$$
q_{0}\left(z_{1}, z_{2}\right)=-\frac{\nu_{z_{2}}}{8 \pi} V_{z_{1}, z_{2}} V_{z_{2}, S} \frac{\mathrm{e}^{\mathrm{i} k r_{z_{2}, S}}}{r_{z_{2}, S}} \beta\left(z_{1}, z_{2}, S\right),
$$

higher order diffraction is generated by iterated applications of the operator (16). As above we define a function $q\left(z_{1}, z_{2}\right)$ that satisfies an equation of the form

$$
q\left(z_{1}, z_{2}\right)=q_{0}\left(z_{1}, z_{2}\right)+\mathcal{I}[q]\left(z_{1}, z_{2}\right)
$$

Once the solution, $q\left(z_{1}, z_{2}\right)$ has been found, the field at the receiver position is evaluated using the propagation operator,

$\mathcal{I}_{\text {prop. }}[q](R):=-\frac{1}{8 \pi} \int_{\Gamma} \int_{\Gamma} q\left(z_{1}, z_{2}\right) \nu_{z_{1}} V_{z_{1}, z_{2}} V_{z_{1}, R} \frac{\mathrm{e}^{\mathrm{i} k r_{z_{1}, z_{2}}}}{r_{z_{1}, z_{2}}} \frac{\mathrm{e}^{\mathrm{i} k r_{R, z_{1}}}}{r_{R, z_{1}}} \beta\left(R, z_{1}, z_{2}\right) d s_{z_{2}} d s_{z_{1}}$,

so that the diffracted field at a receiver position $R$ can be evaluated as the sum of the first order diffracted field and the orders $2, \ldots, \infty$, as in Eq. (15).

\subsection{A special case: Axisymmetric scattering}

The integral equation (14) simplifies significantly in the axisymmetric case, i.e., the case of a circular disk with either a source located at the disk axis, or a plane wave traveling along the disk axis. This is one of the canonical cases 
for which reference solutions are available. Explicit expressions are available for the low-frequency asympotic solution $[19,3,13]$, as well as explicit timedomain expressions derived for first- and second-order diffraction [24]. In $\S 4$ comparisons are made between this new method and the reference solutions for the disk.

Assume the disk is lying in the $x y$-plane, centered at the origin, such that the disk axis is the $z$-axis. In the axisymmetric case the source angles $\theta_{S}$ and $\phi_{S}$ as well as the distance to the source are constant along the perimeter of the disk, see Figure 5 .

The edge of the circle of radius $a$ is parametrized through the function

$$
\gamma(t)=[a \cos (t), a \sin (t)], \quad t \in[0,2 \pi] .
$$

In the following we shall, in a slight abuse of notation, let functions $\beta_{\text {circle }}, q$ and $q_{0}$ of $t$ and $\tau$ represent the functions evalauted in the parametrization. Thus we look for solutions to the equation,

$$
q\left(t_{1}, t_{2}\right)=q_{0}\left(t_{1}, t_{2}\right)-\frac{a}{4 \pi} \int_{0}^{2 \pi} q\left(t_{2}, t\right) \frac{\mathrm{e}^{\mathrm{i} k r_{t_{2}, t}}}{r_{t_{2}, t}} \beta_{\text {circle }}\left(t_{1}, t_{2}, t\right) \mathrm{d} t .
$$

In the axisymmetric case we have two important symmetries of $q$ and $q_{0}$, namely $q\left(t_{1}, t_{2}\right)=q\left(t_{2}, t_{1}\right)$ and $q\left(t_{1}, t_{2}\right)=q\left(t_{1}+t, t_{2}+t\right)$, corresponding to reflection and rotation of the disk respectively. Rotating (19) by $-t_{2}$ and denoting $\tau=t_{1}-t_{2}$,

$$
q(\tau, 0)=q_{0}(\tau, 0)-\frac{a}{4 \pi} \int_{0}^{2 \pi} q\left(0, t-t_{2}\right) \frac{\mathrm{e}^{\mathrm{i} k r_{0, t-t_{2}}}}{r_{0, t-t_{2}}} \beta_{\text {circle }}\left(\tau, 0, t-t_{2}\right) \mathrm{d} t .
$$

Now write $q^{*}(\tau)=q(a, 0)=q(0, \tau)$. Because of the periodicity of the integrand the integral is invariant w.r.t. the change of variables $t \rightarrow t+t_{2}$, thus

$$
q^{*}(\tau)=q_{0}^{*}(\tau)-\frac{a}{4 \pi} \int_{0}^{2 \pi} q^{*}(t) \frac{\mathrm{e}^{\mathrm{i} k r_{0, t}}}{r_{0, t}} \beta_{\text {circle }}(\tau, 0, t) \mathrm{d} t .
$$

With simple trigonometry it can be shown that the observation angle between the tangent at $\gamma(0)$ and the line $(\gamma(0), \gamma(\tau))$ is $\varphi(\tau)=\frac{\tau}{2} \bmod \pi$. Therefore, in the rigid case we have, from equation (12),

$$
\beta_{\text {circle }}(\tau, 0, t)=4 \frac{\sqrt{\sin \frac{\tau}{2} \sin \frac{t}{2}}}{\cos \left(\frac{\tau-t}{4}\right)}, \quad t, \tau \in[0,2 \pi] .
$$


Similarly it can be seen that $r_{0, t}=2 a \sin (t / 2)$, thus the kernel of the integral operator is in this case explicitly known,

$$
q^{*}(\tau)=q_{0}^{*}(\tau)-\frac{1}{\pi} \int_{0}^{2 \pi} q^{*}(t) \frac{\mathrm{e}^{\mathrm{i} k a \sin (t / 2)}}{\cos \left(\frac{\tau-t}{4}\right)} \sqrt{\frac{\sin \frac{\tau}{2}}{\sin \frac{t}{2}}} \mathrm{~d} t .
$$

\section{$4 \quad$ Numerical experiments}

As described in previous sections the solution takes two steps, the first of which is the solution of integral equation (14) or (17), and then the propagation to a receiver position. The full field is evaluated by adding the first order diffracted field and geometrical optics components. A solution is obtained through numerical discretization of the relevant integral equation. In the following tests we will be applying a simple Nyström-trapezoidal method. For the special case of axisymmetric scattering this allows for computing a very accurate solution in a fraction of a second on a standard desktop computer. For more general cases, as for example the scattering by a cube which is demonstrated in the following, more sophisticated methods are likely to be needed for high accuracy.

Although the formulation is arguably a high frequency approach, most of the examples in this section are performed with small wavenumbers. The reason for this is twofold. First, and most important, the formulation would, since it is based on geometrical optics, be expected to have its Achilles Heel in the low-frequency case. Therefore this is the range which should be examined. Secondly, for low frequencies analytic approximations and heuristics are available that we can compare numerical solutions with.

\subsection{Numerical issues}

The primary issue with any numerical method for computing diffraction terms is zone boundaries. Zone boundaries correspond to discontinuities in geometrical optics, and singularities must be expected in the integral representing the first order diffraction at these discontinuities. They are indeed present in the directivity function $\beta$ : When the receiver is placed on a zone boundary, $\beta$, Eq. (3), will have a discontinuity at the point $z$ which determines the shortest path between $S$ and $R$. These singularities can be handled, e.g., by a serial expansion around the singularity as described in [22]. For the integral equation, zone boundaries come into play in two ways: If the source is located in one of the planes containing a face of the scatterer, singularities will appear in the source term of the integral 


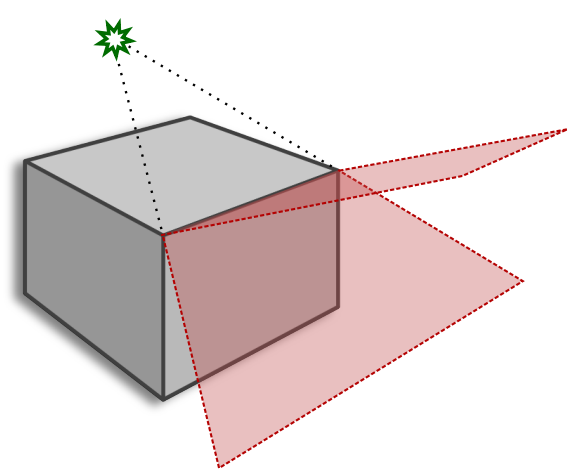

(a)

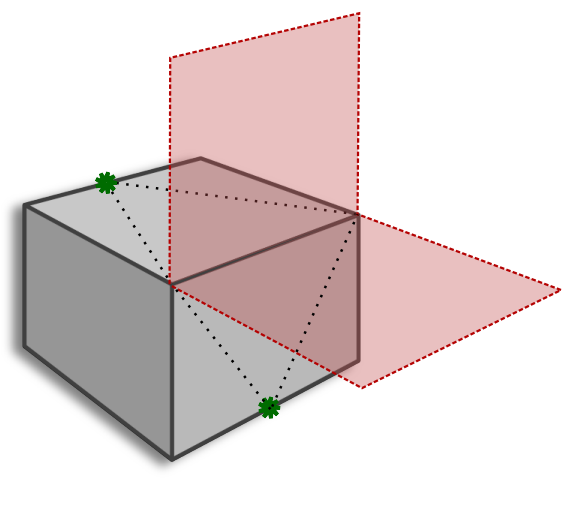

(b)

Figure 6: Illustration of cases where singularities in the $\beta$-factor are encountered for one edge of a cube: (a) The first order diffraction generates two boundary planes. (b) The propagation operator.

equation, $q_{0}\left(z_{1}, z_{2}\right)$, see Figure $6(\mathrm{a})$. The other case is when the receiver is located in one of the same planes, as illustrated in Figure 6(b), which will be manifested as a singularity on the propagation operator. Furthermore, cases where receivers are placed near edges do also require careful numerical treatment, since zone boundaries meet at edges. We shall not discuss these singularities in the present work, but merely point out that these do not, as opposed to the discontinuities in the computation of the first order diffracted field, correspond to discontinuities in the diffracted field. This can readily be seen in the flat plate case, since Eq. (13) has a removable singularity for $\theta_{R}=\pi$.

\subsection{Low frequencies}

The field scattered from an object with Neumann boundary conditions should necessarily display some properties in the low-frequency asymptotic range, which can be checked without direct access to a reference solution. For example, for an incident plane wave, the field directly at the surface should approach that of the free-field incident field, as the frequency goes towards zero. This should be true for any point of the surface, both the illuminated frontal side and the rear side in the shadow. For the special case of axisymmetric scattering, analytic solutions are available.

The low-frequency behavior was evaluated for the axisymmetrical scat- 




(a)



(b)

Figure 7: (a) The computed components of the total field. (b) The relative difference between the field at the center of the surface of the disk and the free field, plotted against the number of discretisation points.

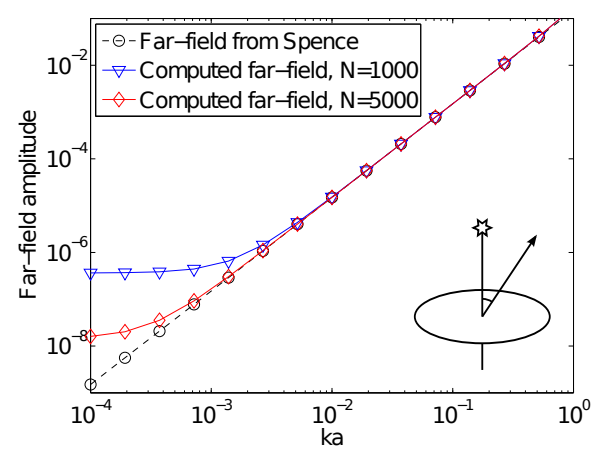

(a)

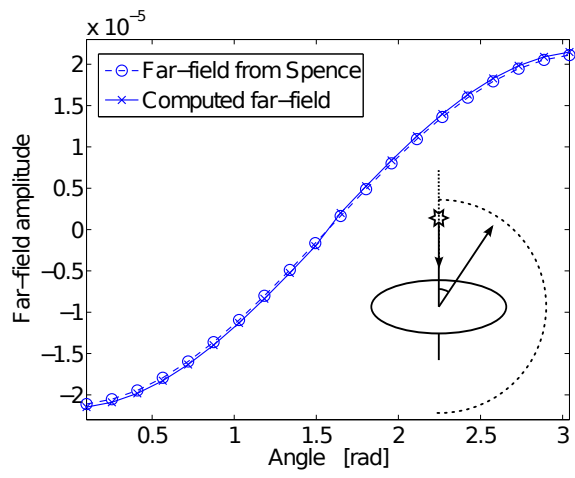

(b)

Figure 8: (a) The absolute difference between computed approximation of the far-field scattered from a circular disk and axisymmetric plane wave incidence in the receiver direction $\pi / 4$ and Spence's asymptotic result. (b) The far-field pattern for $k a=1 / 100$. 




Figure 9: The computed components for the scattering from a cube, $k=0$, normalised to the incident field.

tering from a thin rigid circular disk. A plane-wave travelling along the $z$-axis was used, and the wavefield, $u$, was then computed for a range of receiver positions on the $z$-axis near the plate. The wavenumber can be set to zero, without leading to numerical problems in the computation of the diffraction terms. Furthermore, there are no numerical issues associated with placing receiver positions exactly at the top of the plate. Figure 7 shows the result of this computation. The geometrical-acoustics component has amplitude 2 on the illuminated side $(z>0)$ and 0 on the shadow side $(z<0)$. Notably, we see that the order $2, \ldots, \infty$ order diffraction terms computed from the integral equation formulation add up with geometricalacoustics components and first order diffraction precisely to the incident field. In panel $7 \mathrm{~b}$ ) we see that the discrepancy at the surface of the disk goes to zero with increasing number of discretisation points approximately as $\mathcal{O}\left(N^{-2}\right)$.

For more sophisticated low-frequency asymptotics for the case of the rigid circular disk we consult Spence's classical paper from 1948 [19]. This asymptotic result, which is valid as $|k a| \ll 1$, gives the far-field pattern of the scattering in the case of a normally incident planewave as,

$$
u_{\text {scattered }}^{\infty}(\theta) \sim-\frac{2 a^{2}}{3 \pi} k^{2} \cos \theta,
$$

where $\theta$ denotes the angle of the receiver with the $z$-axis. The far-field 


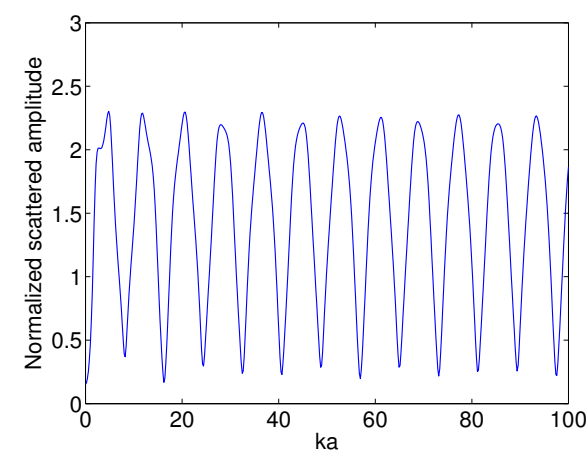

(a)

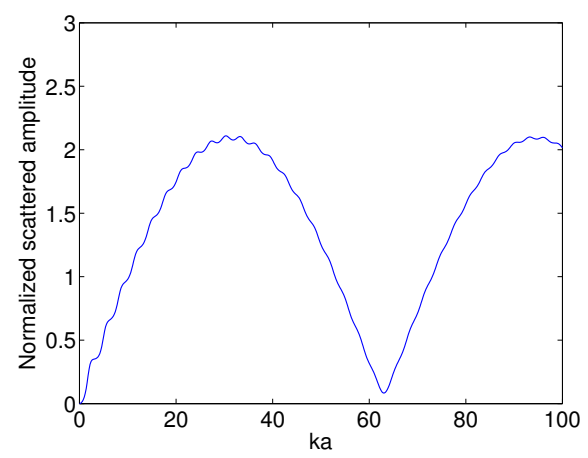

(b)

Figure 10: Axial response for axisymmetric scattering. a) Source and receiver at $z=3, \mathrm{~b}$ ) Source and receiver at $z=10$.

pattern was computed with our method using $N=1000$ and $N=5000$ discretization points, using $u_{\text {scattered }}=u_{\text {diffracted }}$ for observation angles $\neq 0, \pi$. The computed scattered fields show a good correspondence with Spence's result (see Figure 8). It should be noted that a finer discretization is needed the lower the frequency, as can be seen from Figure $8 \mathrm{~b}$ ). However, in this case it is apparent that a modest number of degrees of freedom is sufficient down to very low frequencies; $k \sim 1 / 100$.

As an example of a non-thin scatterer, a cube with side $a=1$ was studied. The cube was placed such that the faces were parallel to the $x-y$, $x-z$, and the $y-z$-planes, with the midpoint of the upper face corresponding to the origin. A point-source was placed at $S=[1,1,1] \cdot 10^{5} / \sqrt{3}$, in which case zone boundary problems only occur in the computation of the first order diffracted field. Using a Nyström method the solution to the integral equation was computed, and the field was evaluated along the $z$-axis. The result of this experiment can be seen in Figure 9. It is clear that, as in the case of the circle, that the computed diffracted fields add up to produce the incident field, as should be expected.

Further numerical examples involving low-frequency behavior for cubes and square plates can be found in [21].

\subsection{Higher frequencies}

An approximation for the scattered field has been presented by Keiffer et al. in [10] for high frequencies in the case of axisymmetric source. We 


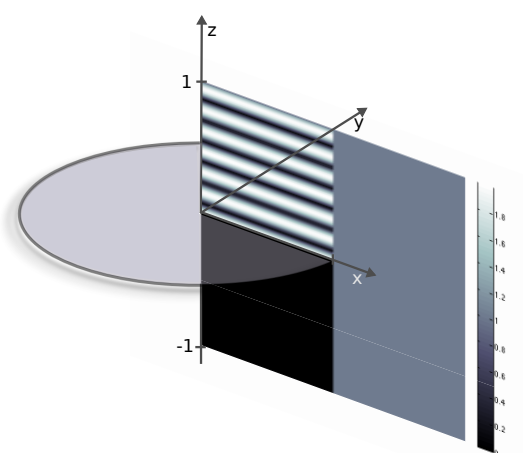

(a)



(b)



(c)

Figure 11: Computing the scattered field from a disk of radius $a=1$, wavenumber $k=20$. Absolute field strengths: a) Geometrical optics, b) Diffracted, c) Total. 
computed the scattered field for the frequency range of $k a \in[0,100]$, using $N=1000$ discretization points, and results are presented in Fig. 10. Excellent agreement can be observed upon inspection of Fig. 4 and 6 in Ref. $[10]$.

Finally, we demonstrate that the approach indeed gives a qualitatively correct field, i.e., without the discontinuities of geometrical optics. Setting $k=20$, we computed the solution for the axisymmetric scattering from a disk, $a=1$, for a section of $3 \mathrm{D}$ space. The result of this computation can be seen in Figure 11, where it is apparent that the discontinuity of the geometrical optics approximation is canceled by the discontinuity of the diffracted field.

Although no attempts have yet been made to speed up computations for higher frequencies, which is outside the scope of this work, we believe that this new formulation provides a new platform for attempts at high frequency scattering problems.

\section{References}

[1] A. Asheim And D. Huybrechs, Local solutions to high-frequency 2D scattering problems, J. Comput. Phys., 229 (2010), pp. 5357-5372.

[2] M. A. Biot And I. Tolstoy, Formulation of wave propagation in infinite media by normal coordinates with an application to diffraction, J. Acoust. Soc. Am., 29 (1957), pp. 381-391.

[3] J. J. Bowman, T. B. A. Senior, and P. L. E. Uslenghi, Electromagnetic and acoustic scattering by simple shapes (revised edition), New York, Hemisphere Publishing Corp., 1 (1987).

[4] O. P. Bruno, C. Geuzaine, J. A. Monro Jr, and F. Reitich, Prescribed error tolerances within fixed computational times for scattering problems of arbitrarily high frequency: the convex case, Phil. Trans. R. Soc. A, 362 (2004), pp. 629-645.

[5] S. N. Chandler-Wilde And S. Langdon, A Galerkin boundary element method for high frequency scattering by convex polygons, SIAM J. Numer. Anal., 45 (2007), pp. 610-640.

[6] V. Dominguez and I. G. Graham, A hybrid numerical-asymptotic boundary integral method for high-frequency acoustic scattering, Numer. Math., 106 (2007), pp. 471-510. 
[7] D. Huybrechs and S. Vandewalle, A sparse discretisation for integral equation formulations of high frequency scattering problems, SIAM J. Sci. Comput., 6 (2007), pp. 2305-2328.

[8] J. T. KAJIYA, The rendering equation, ACM SIGGRAPH, 20 (1986), pp. $143-150$.

[9] T. KaWAI, Sound diffraction by a many-sided barrier or pillar, J. Sound Vib., 79 (1981), pp. 229-242.

[10] R. S. Keiffer, J. C. Novarini, and G. V. Norton, The impulse response of an aperture: Numerical calculations within the framework of the wedge assemblage method, J. Acoust. Soc. Am., 95 (1994), pp. 3-12.

[11] J. B. Keller, A geometrical theory of diffraction, J. Opt. Soc. Am., 52 (1962), pp. 116-130.

[12] R. G. Kouyoumuian and P. H. Pathak, A uniform geometrical theory of diffraction for an edge in a perfectly conducting surface, Proc. of the IEEE, 62 (1974), pp. 1448-1461.

[13] G. Kristensson and P. C. Waterman, The T matrix for acoustic and electromagnetic scattering by circular disks, J. Acoust. Soc. Am., 72 (1982), pp. 1612-1625.

[14] H. M. Macdonald, A class of diffraction problems, P. Lond. Math. Soc., 2 (1915), pp. 410-427.

[15] H. Medwin, E. Childs, And G. M. Jebsen, Impulse studies of double diffraction: A discrete Huygens interpretation, J. Acoust. Soc. Am., 72 (1982), pp. 1005-1013.

[16] A. D. PIERCE, Diffraction of sound around corners and over wide barriers, J. Acoust. Soc. Am., 55 (1974), pp. 941-955.

[17] S. L. S N Chandler-Wilde, I G Graham and E. A. Spence, Numerical-asymptotic boundary integral methods in high frequency acoustic scattering, Acta Numer., (to appear 2012).

[18] R. W. Scharstein And A. M. J. Davis, Time-domain threedimensional diffraction by the isorefractive wedge, IEEE Trans. Antennas Propag., 46 (1998), pp. 1148-1158.

[19] R. D. Spence, The diffraction of sound by circular disks and apertures, J. Acoust. Soc. Am., 20 (1948), pp. 380-386. 
[20] J. E. Summers, Reverberant acoustic energy in auditoria that comprise systems of coupled rooms, $\mathrm{PhD}$ thesis, Rensselaer Polytechnic Institute, Troy, NY, USA, 2003.

[21] U. P. Svensson And A. Asheim, Edge-diffraction based integral equation for the scattering from convex rigid polyhedra, in Proc. of Forum Acusticum 2011, Aalborg, Denmark, 27 June - 1 July 2011, pp. 179 184 .

[22] U. P. Svensson and P. T. Calamia, Edge-diffraction impulse responses near specular-zone and shadow-zone boundaries, Acta Acustica with Acustica, 92 (2006), pp. 501-512.

[23] U. P. Svensson, P. T. Calamia, and S. Nakanishi, Frequencydomain edge diffraction for finite and infinite edges, Acta Acustica with Acustica, 95 (2009), pp. 568-572.

[24] U. P. Svensson, R. I. Fred, And J. Vanderkooy, An analytic secondary source model of edge diffraction impulse responses, J. Acoust. Soc. Am., 106 (1999), pp. 2331-2344.

[25] J. Vanderkooy, A simple theory of cabinet edge diffraction, J. Audio. Eng. Soc., 39 (1991), pp. 923-933. 